\title{
Development of Diazaborines as ROS Sensitive Linkers for the Construction of Stimuli- Responsive Antibody Drug Conjugates
}

João P. M. António, ${ }^{1}$ Joana Inês Carvalho, ${ }^{1}$ Ana S. André, ${ }^{2}$ Joana N. R. Dias, ${ }^{2}$ Hélio Faustino, ${ }^{1}$ Ricardo M. R. M. Lopes, ${ }^{1}$ Luis F. Veiros,${ }^{3}$ Gonçalo J. L. Bernardes, ${ }^{4,5}$ Frederico A. da Silva, ${ }^{2}$ Pedro M. P. Gois ${ }^{1 *}$.

${ }^{1}$ Research Institute for Medicines (iMed.ULisboa), Faculdade de Farmácia, Universidade de Lisboa, Lisboa, Portugal

${ }^{2}$ Centro de Investigação Interdisciplinar em Sanidade Animal, Faculdade de Medicina Veterinária, Universidade de Lisboa, Lisboa, Portugal

${ }^{3}$ Centro de Química Estrutural and Departamento de Engenharia Química, Instituto Superior Técnico, Universidade de Lisboa, Portugal

${ }^{4}$ Instituto de Medicina Molecular João Lobo Antunes, Faculdade de Medicina, Universidade de Lisboa, Avenida Professor Egas Moniz, 1649-028 Lisboa, Portugal.

${ }^{5}$ Yusuf Hamied Department of Chemistry, University of Cambridge, Lensfield Road, CB2 1EW, Cambridge, United Kingdom.

* These authors contributed equally to the work.

KEYWORDS: Antibody-drug conjugate, Boronic acids, Diazaborines, Oxidative stress, Cleavable linkers, ROS.

ABSTRACT: Antibody-drug conjugates (ADCs) are a new class of therapeutics that combine the lethality of potent cytotoxic drugs with the targeting ability of antibodies to selectively deliver drugs to cancer cells. The synthesis of ADCs is challenging, and studies in this area show that their therapeutic effect is highly dependent on the chemistries used to connect both functions. Therefore, the linker evolved in recent years from being a simple chemical spacer to a functional structure that controls the potency and selectivity of ADCs. The linker provides a platform to integrate mechanisms to access 
synthetic homogeneity, stability in circulation and more importantly the installation of chemical units that release the drug as a response to the disease chemical environment. In this study we show for the first time the synthesis of a reactive-oxygen-species (ROS) responsive ADC (VL-DAB31-SN-38) that is highly selective and cytotoxic to B-cell lymphoma (CLBL-1 cell line, $\mathrm{IC}_{50}$ value of $54.1 \mathrm{nM}$ ). The synthesis of this ADC was possible due to the discovery that diazaborines (DABs) are a very effective ROS responsive unit $\left(0.422\right.$ and $0.103 \mathrm{M}^{-1} \mathrm{~S}^{-1}$ with 100 and 10 equiv. of $\mathrm{H}_{2} \mathrm{O}_{2}$ respectively), that is also very stable in buffer (over 14 days at different pHs) and in plasma (over 5 days). DFT calculation performed on this system revealed a favourable energetic profile ( $\Delta \mathrm{GR}=-74.3 \mathrm{kcal} / \mathrm{mol})$ similar to the oxidation mechanism of aromatic boronic acids. DABs very fast formation rate and modularity enabled the construction of different ROS responsive linkers featuring self-immolative modules, bioorthogonal functions and bioconjugation handles. These structures were used in the site-selective functionalization of a VL antibody and in the construction of the homogeneous ADC. The enclosed ROS-responsive linker technology based on DABs, is expected to become a valuable tool to prepare stimuli responsive therapeutic materials, as ROS is a very important hallmark in several important diseases.

\section{Introduction}

In recent years, reactive oxygen species (ROS) gained considerable recognition because of their central role in cellular homeostasis through the regulation of numerous signaling pathways. ${ }^{1}$ This family of signaling molecules is responsible for, among others mechanisms, controlling DNA transcription and regulating cell proliferation and differentiation. The vast majority of cellular $\operatorname{ROS}(\approx 80 \%)$ is generated during the oxidative phosphorylation in the electron transport chain of mitochondria, with the remaining part being produced in peroxisomes and endoplasmic reticulum. ${ }^{2}$ In high levels, ROS are harmful to cells as they can damage proteins, lipids and DNA, ultimately leading to metabolic disfunction and cell death. ${ }^{3}$ Thus, cells have built-in mechanisms to counterbalance the increase of ROS levels which include a complex scavenging system based on glutathione (GSH) and redox enzymes such as

superoxide dismutase, glutathione peroxidase, glutathione reductase, thioredoxin and catalase.$^{3-5}$ The preservation of this tenuous redox balance is essential for maintaining cellular homeostasis. 
Contrary to healthy cells, cancer cells, due to a defective mitochondrial oxidative metabolism, have an inherently high level of ROS which is indispensable to sustain the biochemical alterations required for the initiation, promotion and progression of the disease.$^{6}$ The elevated oxidative stress in cancer cells, when compared with healthy cells, has been explored as a trigger to promote the selective delivery of cytotoxic and imaging payloads. ${ }^{7-9}$ These drug delivery systems are generally based on nanoparticles and polymeric supramolecular constructs bearing organochalcogen, thioether, thioketal or aryloxalate linkages that, when exposed to ROS, promote the payload release upon changing their physical properties. ${ }^{7}$ Interestingly, although this strategy has been used to successfully promote the drug delivery of different nanosystems, to the best of our knowledge, these linkages have not been used in the construction of well-defined ROS-responsive targeting drug conjugates like ADCs. This lack of use is probable related with the fact that these functions endure the physiologic conditions more effectively when incorporated in a material's supramolecular structure rather than when solved exposed in the form of a bioconjugate linker. Therefore, the engineering of innovative architectures of well-defined ROSresponsive targeting drug conjugates relies on the discovery of chemical functions that sense diseaseassociated concentrations of ROS, while maintaining the structural integrity of the bioconjugate in circulation.

Aromatic boronic acids (BAs) are well-known for being readily oxidized to the corresponding phenols in the presence of various ROS, and this unique mechanism has been extensively explored in the design of multiple functional materials for drug delivery (Fig. 1a). ${ }^{10,11}$ However, aromatic BAs also exhibit poor stability under physiologic conditions and a promiscuous reactivity with endogenous molecules, such as vicinal diols or proteins, which often translates into poor pharmacokinetic and toxicity profiles. ${ }^{12,13}$ Therefore, despite very favorable oxidation kinetics in the presence of ROS, the instability and off-target reactivity of BAs constitute a major obstacle for the use of this function in the design of ROS-responsive linkers for targeted drug conjugates. 
<smiles>[R1]C(=O)c1ccccc1B(O)O</smiles><smiles>Oc1ccccc1</smiles>

Readily oxidized by ROS

Used for decaging of drugs and probes

Limited by instability and off-target reactivity

\section{c. This studv}
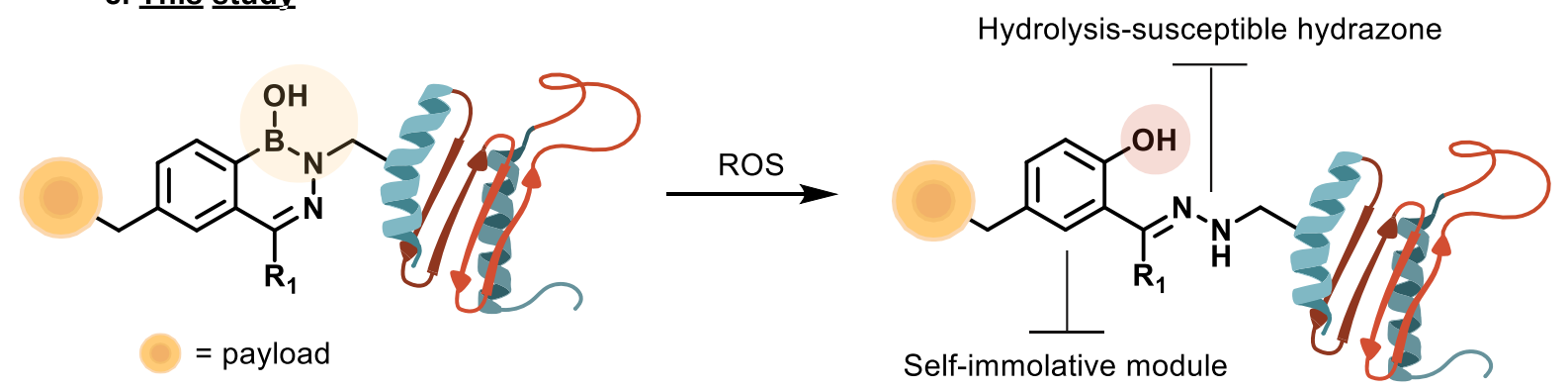

Well-defined ROS responsive linker

Improved stability, compatible with bioconjugation, sensitivity to ROS

Figure 1 | Proposed structure for ROS-responsive linker based on DAB scaffold. (a) Aromatic boronic acids oxidation by ROS; (b) Diazaborines as bioorthogonal bioconjugation tool; (c) This study: Diazaborines oxidation by ROS and its application as ROS-responsive linkers for targeting drug conjugates. ROS, reactive species of oxygen.

In the early 80 s, diazaborines (DABs) emerged as a promising class of boronated heterocycles with antibacterial properties. ${ }^{14-17}$ More recently, the very efficient formation of DABs under bioconjugation conditions, established this reaction as a powerful click-type transformation in chemical biology. ${ }^{18,19}$ In DABs, the BA function is inserted in a B-N heterocycle where the boron vacant orbital is stabilized by the lone pair of electrons of the adjacent nitrogen (Fig. 1b). ${ }^{12,16,20}$ Despite this, as corroborated by recent studies, this architecture does not suppress the Lewis acidity of the boron centre. ${ }^{21}$ Therefore, we envisioned that, if DAB's boron center retains the BA's oxidative sensitivity in the presence ROS, the improved stability of this scaffold could contribute to bridge the technological gap that limits the 
construction of well-defined ROS-responsive linker for targeting drug conjugates. The demonstration of this hypothesis constitutes the main focus of this study (Fig. 1c).

\section{Results and Discussion}

Depending on the stereo-electronic properties of the hydrazine and 2-carbonylphenyl BA components, DABs with different stabilities under aqueous media can be obtained as a consequence of the equilibrium between the close and the open forms. ${ }^{20}$ In the open form, the BA function is exposed and the hydrazone linkage is expected to be more prone to hydrolysis. Therefore, we initiated this study by evaluating the influence of different substituents in the formation and stability of these heterocycles. Hence a panel of DABs was synthesized by mixing in water 2-formylphenyl BA (2-FPBA) or 2-acetyl phenyl BA (2-APBA) with hydrazines featuring different $N$-substituents (Fig. 2a). 
a.<smiles>[R2]NNN</smiles>

$\mathrm{R}_{1}=\mathrm{H} \quad$ 2-FPBA

$R_{1}=$ Me 2-APBA

Formation rate<smiles>OB1c2ccccc2C=NN1c1ccccc1</smiles>

1

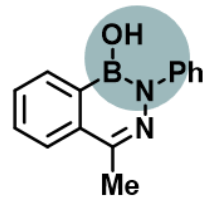

5

b.

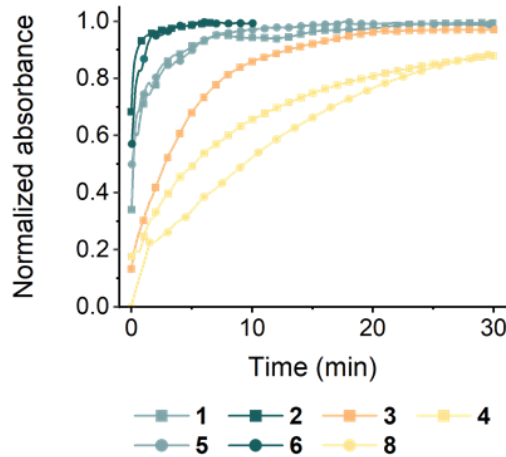

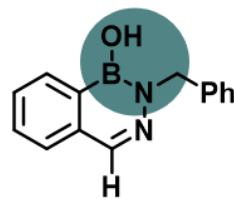

2

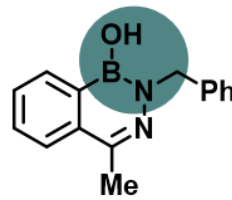

6<smiles>O=C(c1ccccc1)N1N=Cc2ccccc2B1O</smiles>

3

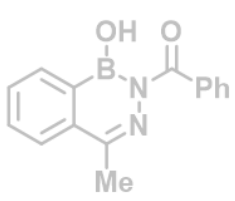

7<smiles>OB1c2ccccc2C=NN1[AsH3-]</smiles>

4<smiles>[Y5]N1N=C(C)c2ccccc2B1O</smiles>

8

c.

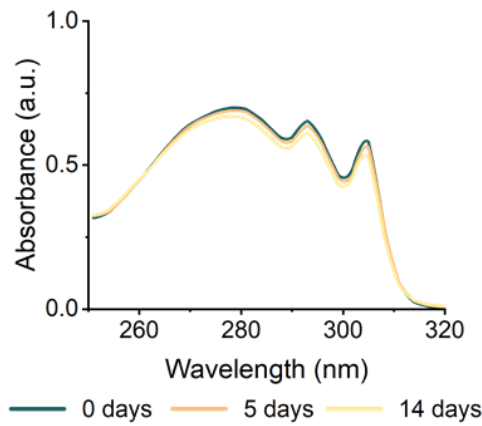

Figure 2 | Diazaborines 1-8 and their stability. (a) Diazaborines prepared from the reaction of phenyl, benzyl, benzoyl and tosyl hydrazines with 2-FPBA and 2-ABPA; (b) Normalized formation kinetics of DABs 1-8 (7 was excluded due to unreliable results); (c) UV-Vis spectra of compound 2 after 5 and 14 days.

Once prepared, DABs 1-8 were tested for their stability in ammonium acetate at $\mathrm{pH}$ 7.4. As shown in previous studies, also in this assay, DABs 2-4, prepared from 2-FPBA proved to be considerably more stable than those prepared from 2-APBA (5-8), as no degradation was observed for these compounds over 14 days (Fig. 2c and Supplementary Fig. S5). Despite being prepared from 2-FBPA, compound 1 displayed a poor stability which is possibly related with the low stability of the phenyl hydrazine in these conditions. 
The reaction of hydrazines with 2-carbonylphenyl BA was shown to exhibit very fast kinetics and have been used recently as a click-type reaction to assemble bioconjugates. ${ }^{19,22}$ Therefore, we studied the formation kinetics of DABs 1-8 (7 was excluded from this study due to unreliable results possibly related to possibly due to the already reported equilibrium of open and closed forms and the formation of dimers). ${ }^{23}$ As shown in Fig. 2b, in ammonium acetate $\mathrm{pH} 7.4$ (final concentration $60 \mu \mathrm{M}$ ), the reaction of benzyl hydrazine with 2-FPBA and 2-ABPA displayed the fastest kinetics, generating DABs 2 and $\mathbf{6}$ in less than $10 \mathrm{~min}$, while hydrazines with electron withdrawing $N$-substituents were slightly slower, affording the respective DABs in up to $1 \mathrm{~h}$. Interestingly, pairs bearing the same $N$-substituent appear to display similar reaction rates, implying that the stereo-electronic properties of the hydrazine substituent are essential to the reaction kinetics.

Considering these results, DABs featuring alkylic $N$-substituents like DAB 2 and $\mathbf{6}$ displayed the best performance in terms of stability in aqueous conditions and kinetics of formation. Therefore, we next tested if DABs retained the ability to be oxidized in the presence of ROS. Hence, a $50 \mu \mathrm{M}$ solution of 2 was incubated with 100 equiv. of $\mathrm{H}_{2} \mathrm{O}_{2}$ (ammonium acetate $20 \mathrm{mM} \mathrm{pH}$ 7.4). After 30 min, the mixture was analyzed by Electrospray Ionization-Mass Spectrometry (ESI-MS) and DAB 2 generated the corresponding salicyl-hydrazone 9 (Fig. 3). Similar oxidations were observed for the remaining DABs 1-8 under these conditions (Supplementary Fig. S8).

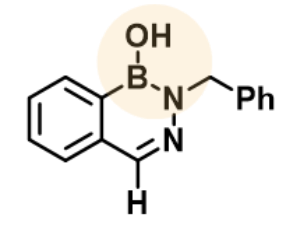

2 $\mathrm{m} / \mathrm{z}[\mathrm{M}+\mathrm{H}]^{+}=237.1$

$$
\underset{30 \text { min. }}{\stackrel{\mathrm{H}_{2} \mathrm{O}_{2} \text { (100 equiv.) }}{\underset{\mathrm{NH}_{4} \mathrm{CH}_{3} \mathrm{CO}_{2}, \mathrm{pH}}{\longrightarrow}}}
$$

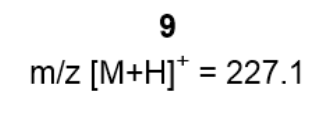

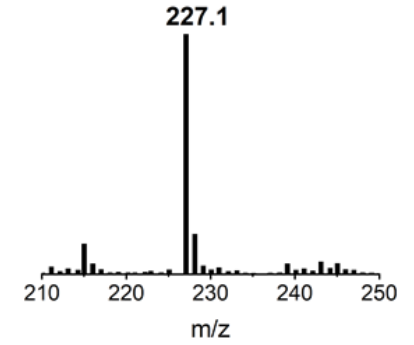

Figure 3 | ESI-MS spectrum of DAB 2 oxidation after $30 \mathrm{~min}$ in the presence of 100 equiv. of $\mathrm{H}_{2} \mathrm{O}_{2}$ peroxide. Compound $2(10 \mathrm{mM}, \mathrm{DMF})$ was diluted in ammonium acetate solution $20 \mathrm{Mm}$ at $\mathrm{pH} 7.4$ to a final concentration of $50 \mu \mathrm{M}$ in the presence of 100 equiv. of $\mathrm{H}_{2} \mathrm{O}_{2}$. The mixture was stirred at $25^{\circ} \mathrm{C}$ for 30 minutes and then analysed by ESI-MS to observe the full conversion into the corresponding salicyl-hydrazone 9. 
Encouraged by these results, the aqueous stability of DAB 2 was further tested in more demanding conditions, namely at different pHs and in human plasma. Hence, DAB $2(100 \mu \mathrm{M})$ was incubated in at $\mathrm{pH} 4.5$ (acetate buffer $50 \mathrm{mM}), 7.4(\mathrm{KPi}$ buffer $50 \mathrm{mM}$ ) and 9.0 (carbonate buffer $50 \mathrm{mM}$ ) and the stability was monitored by High Performance Liquid Chromatography (HPLC) at different time points. As shown in Fig. 4a, DABs concentration remained constant after 14 days in all three conditions. Similar results were obtained upon incubation of DAB 2 in human plasma at $37^{\circ} \mathrm{C}(120 \mu \mathrm{M})$, with no visible degradation after 5 days.

Pleased with its stability, we decided to study the oxidation process in detail. We incubated DAB 2 with 100 equiv. of $\mathrm{H}_{2} \mathrm{O}_{2}$ at pH 7.4 (ammonium acetate $20 \mathrm{mM}$ ) and followed the disappearance of the starting material by HPLC. As observed in Fig. 4b, at $100 \mu \mathrm{M}$ the starting material was swiftly oxidized, with a calculated oxidation half-life around $15 \mathrm{~min}$. Proportional results were obtained when repeating the experiment with $50 \mu \mathrm{M}, 500 \mu \mathrm{M}$ and $1 \mathrm{mM}$ of starting material which enabled the determination of the reaction rate as $0.422 \mathrm{M}^{-1} \mathrm{~s}^{-1}$ (Supplementary Fig. S9 and S11).

Although useful in our preliminary understanding of the reaction mechanism, these results with $10 \mathrm{mM}$ $\mathrm{H}_{2} \mathrm{O}_{2}$ have limited relevance in terms of biological applications. Hence, we proceeded to evaluate the oxidation profile of DAB 2 in conditions that are closer to those associated with the physiopathological conditions of tumor cells. Therefore, the oxidation of 2 was performed using only 10 equiv. of $\mathrm{H}_{2} \mathrm{O}_{2}$. As expected, by reducing the oxidant concentration the reaction rate is slightly decreased. Nevertheless, at $100 \mu \mathrm{M}$ of DAB 2 the reaction proceeds with an oxidation half-life of $3.5 \mathrm{~h}$ (Fig. $4 \mathrm{c}$ ). When repeating the experiment at $50 \mu \mathrm{M}, 500 \mu \mathrm{M}$ and $1 \mathrm{mM}$ of DAB 2, a reaction rate of $0.103 \mathrm{M}^{-1} \mathrm{~s}^{-1}$ was calculated (Supplementary Fig. S10 and S11). 
a.

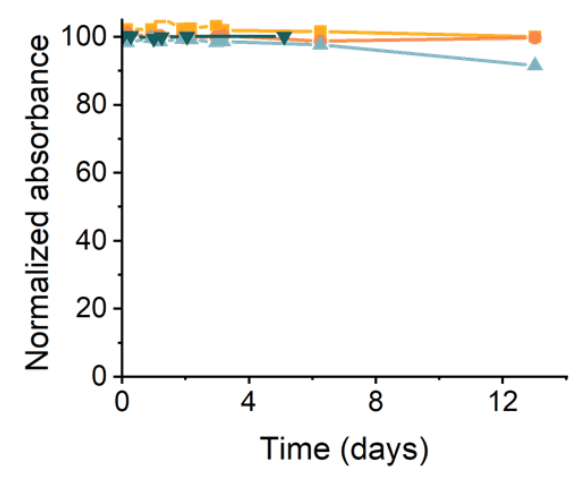

-2 in $\mathrm{pH} 4.5-0-2$ in $\mathrm{pH} 7.4$

$-\mathbf{-} \mathbf{2}$ in $\mathrm{pH} 9.0-\boldsymbol{\nabla}-\mathbf{2}$ in Plasma

c.

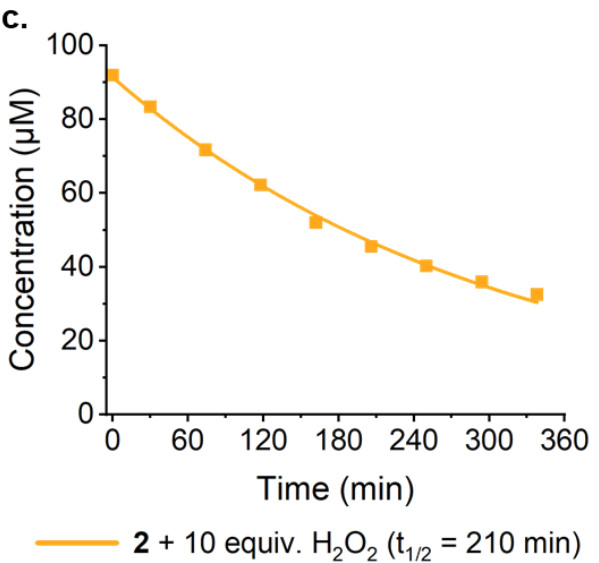

b.

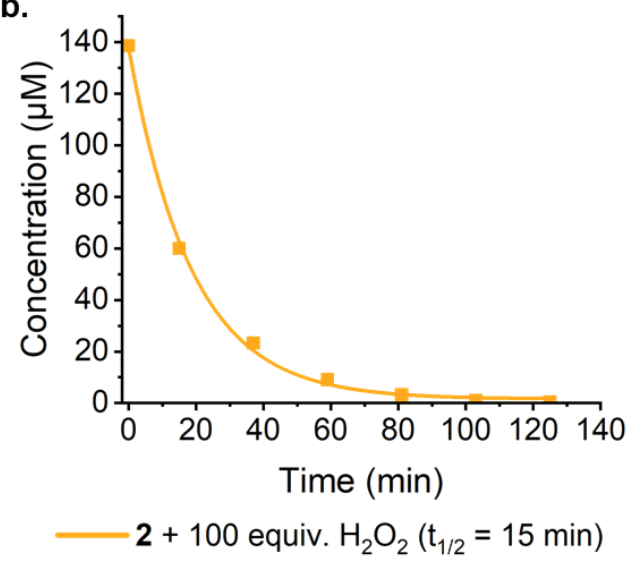

d.

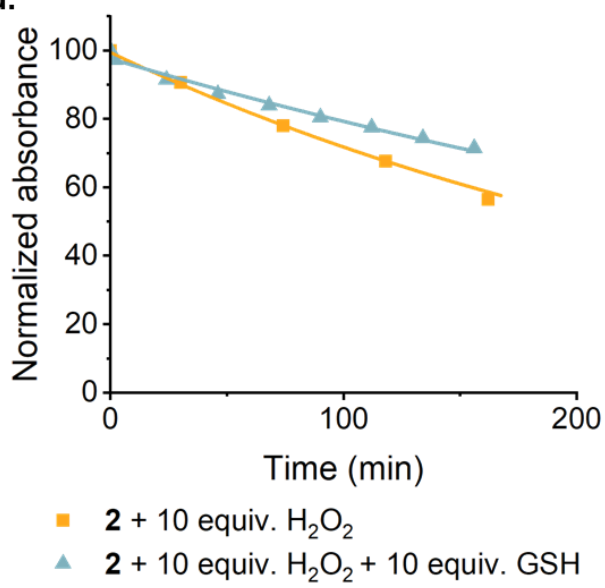

Figure 4 | Stability of DAB 2 at different conditions. (a) Stability of DAB 2 at pH 4.5, 7.4, 9.0 and in plasma; (b) Oxidation profile of DAB 2 in the presence of 100 equiv. $\mathrm{H}_{2} \mathrm{O}_{2}$; and (c) 10 equiv. $\mathrm{H}_{2} \mathrm{O}_{2}$; (d) Oxidation profile of DAB 2 in the presence and absence of glutathione. DAB, diazaborine; GSH, glutathione.

Although ROS plays a fundamental role in tumoral proliferation, the increased intracellular concentration of ROS can be noxious for tumor cells if not adequately counterbalanced. The major redox balancing mechanism in tumor cells encompasses an increase in GSH concentration and this delicate equilibrium is essential to maintain the redox homeostasis of cancer cells. Therefore, we questioned if $\mathrm{H}_{2} \mathrm{O}_{2}$ would still be able to oxidize DABs in the presence of high concentrations of GSH. To simulate cancer cell conditions, DAB $2(100 \mu \mathrm{M})$ was incubated with 10 equiv. of GSH (1 mM), followed by the addition of 10 equiv. of $\mathrm{H}_{2} \mathrm{O}_{2}(1 \mathrm{mM})$. As expected, although the reaction in the presence of GSH is slightly slower (Fig. 4d), importantly, the oxidation mechanism is still operative.

Next, we investigated the influence of the $\mathrm{pH}$ in the oxidation rate. Accordingly, DAB $2(100 \mu \mathrm{M})$ was incubated with 100 and 10 equiv. of $\mathrm{H}_{2} \mathrm{O}_{2}$ in carbonate buffer $50 \mathrm{mM}$ at $\mathrm{pH} 9.0$. Under these conditions 
the oxidation was clearly accelerated, proceeding with half-lives of 5 and 14 min, respectively (Fig. 5ab). Inversely, when the assay was performed in acetate buffer $50 \mathrm{mM}$ at $\mathrm{pH} 4.5$, the oxidation was completely inhibited, even in the presence of 100 equiv. of oxidizing agent (Fig. 5c-d).

a.

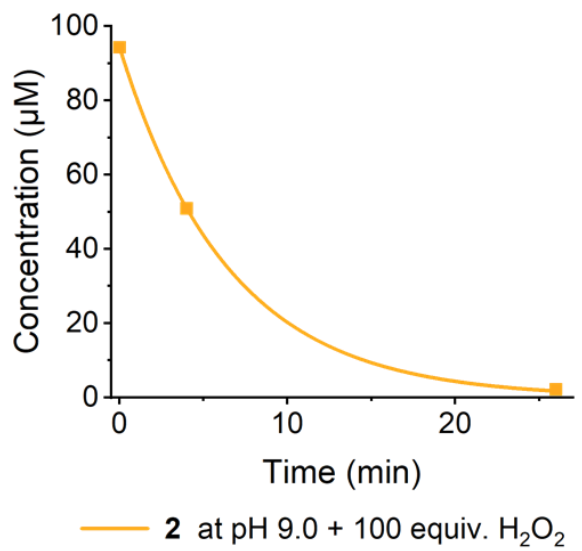

c.

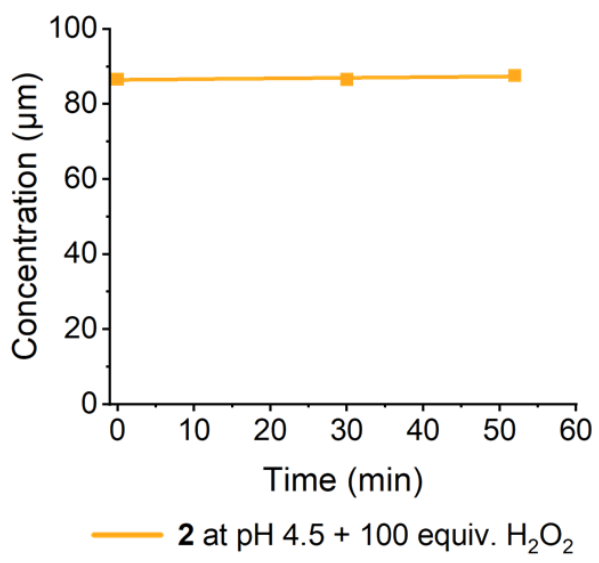

b.

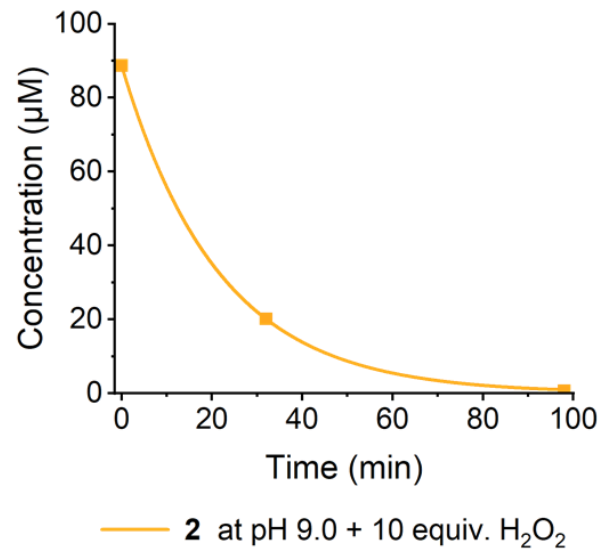

d.

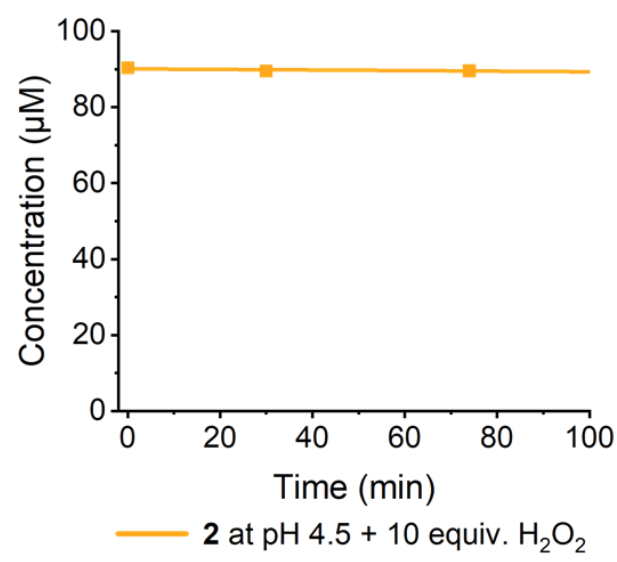

Figure 5 | Oxidation profile of DAB 2 at pH 9.0 or 4.5. At pH 9 in the presence of (a) 100 equiv. and (b) 10 equiv. $\mathrm{H}_{2} \mathrm{O}_{2}$; At pH 4.5 in the presence of (c) 100 equiv. and (d) 10 equiv. $\mathrm{H}_{2} \mathrm{O}_{2}$.

To rationalize some of the aforementioned observations, we studied the oxidation mechanism of DAB 2 in the presence of $\mathrm{H}_{2} \mathrm{O}_{2}$ by means of DFT calculations. ${ }^{24}$ The result is depicted in a simplified way in Fig. 6, showing the more relevant steps along the path. The detailed profile is presented as Supporting Information (Supplementary Fig. S36).

The mechanism starts with nucleophilic attack of the peroxide O-atom to the boron atom of the DAB molecule, overcoming a barrier of $23.1 \mathrm{kcal} / \mathrm{mol}$. The next relevant step is the aryl transposition from the boron to the $\mathrm{O}$-atom with an associated barrier of $\Delta \mathrm{G}^{\ddagger}=28.2 \mathrm{kcal} / \mathrm{mol}$. This is the highest barrier of the entire mechanism $\left(\mathbf{T S}_{\mathbf{C D}}\right)$ and corresponds to the overall energy barrier of the reaction. The path 
proceeds with nucleophilic attack of a water molecule and $\mathrm{B}-\mathrm{N}$ bond breaking and, finally, there is hydrolysis of the $\mathrm{O}-\mathrm{B}$ bond by a second water molecule, producing the final phenol group and boric acid. The reaction is thermodynamically favorable with an overall free energy balance of $\Delta \mathrm{G}_{\mathrm{R}}=-74.3$ $\mathrm{kcal} / \mathrm{mol}$. The mechanism calculated allows a rationalization of the observed $\mathrm{pH}$ influence on the reaction rate. Accordingly, under acidic conditions the $\mathrm{H}_{2} \mathrm{O}_{2}$ will be mostly protonated and, consequently, unable to attack the boron, whereas in basic medium its nucleophilicity is increased, facilitating the attack that is the starting point of the reaction. ${ }^{25,26}$ The equivalent mechanism with phenyl BA as substrate, was also calculated for comparison purposes (Supplementary Fig. S37). These results indicate a slightly more facile reaction than the one involving DAB 2 , with a lower overall barrier $\left(\Delta \mathrm{G}^{*}\right.$ $=26.1 \mathrm{kcal} / \mathrm{mol})$ as well as a more favorable free energy balance $\left(\Delta \mathrm{G}_{\mathrm{R}}=-91.9 \mathrm{kcal} / \mathrm{mol}\right)$. However, the difference is quite small and clearly support the observed oxidation of DABs.

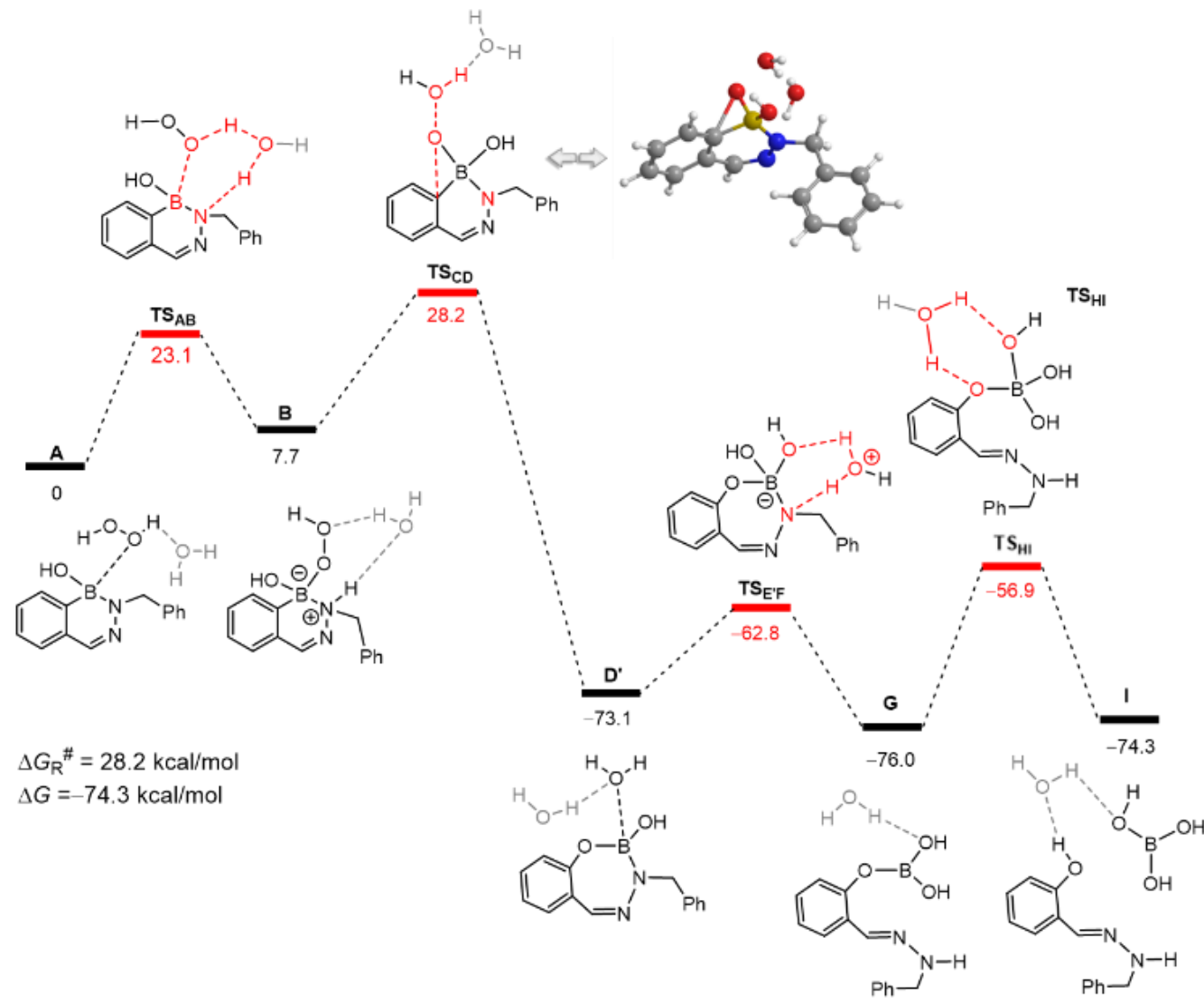

Figure 6 | Simplified free energy profile calculated for the oxidation of DAB 2 by $\mathrm{H}_{2} \mathrm{O}_{2}$ (most relevant steps). Free energy values $(\mathrm{kcal} / \mathrm{mol})$ relative to the initial reactants A. DFT calculations at the M06-2X/6-311G $(\mathrm{d}, \mathrm{p})$ level were performed using the GAUSSIAN 09 package. The model used in the calculations included an explicit water molecule and solvent effects (water) were further considered by means of the PCM model. A complete account of the computational details and the corresponding list of references are provided as SI. 
Once established that alkylic-derived DABs are a valuable scaffold to design ROS-responsive linkers, we initiated a study to integrate this responsive moiety in the structure of a functional bioconjugate. Considering that, upon DAB oxidation a hydrolysis-susceptive hydrazone is generated, we first designed a linker in which the biomolecule is attached to the 2-FPBA component through a maleimide, while the functional payload is linked to the hydrazine. Therefore, the 2-FPBA was modified to install a maleimide function over 6 synthetic steps starting from 10 (Fig. 7a and Supporting Information). Once prepared, cross-linker 11 was reacted with benzyl hydrazine to generate DAB 12, which was evaluated in the functionalization of a model peptide featuring a cysteine (Cys) residue. As shown in Fig. 7, the bioconjugation with laminin fragment effectively afforded bioconjugate $\mathbf{1 3}$ which was successfully oxidized with $\mathrm{H}_{2} \mathrm{O}_{2}$ overnight (Fig. 7b-c).

a.

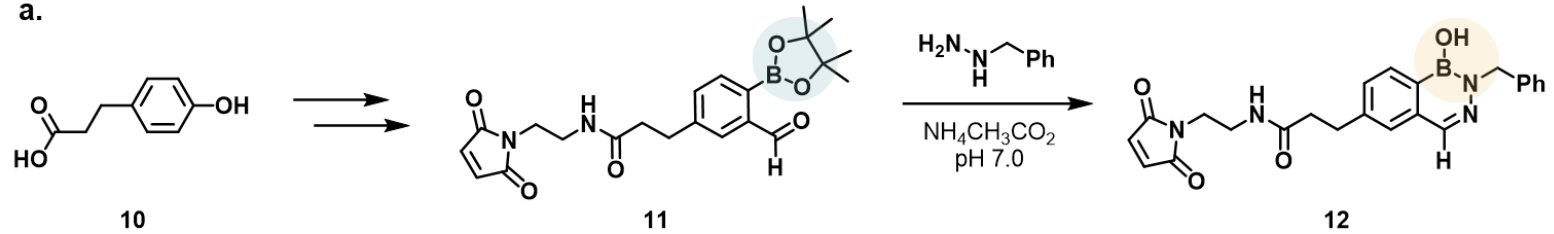

b.

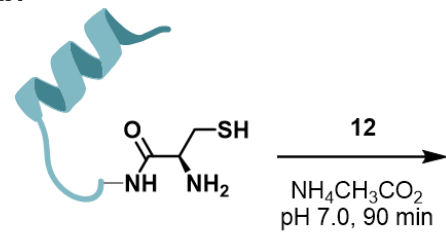

Laminin fragment $\mathrm{m} / \mathrm{z}[\mathrm{M}+\mathrm{H}]^{+}=967.4$
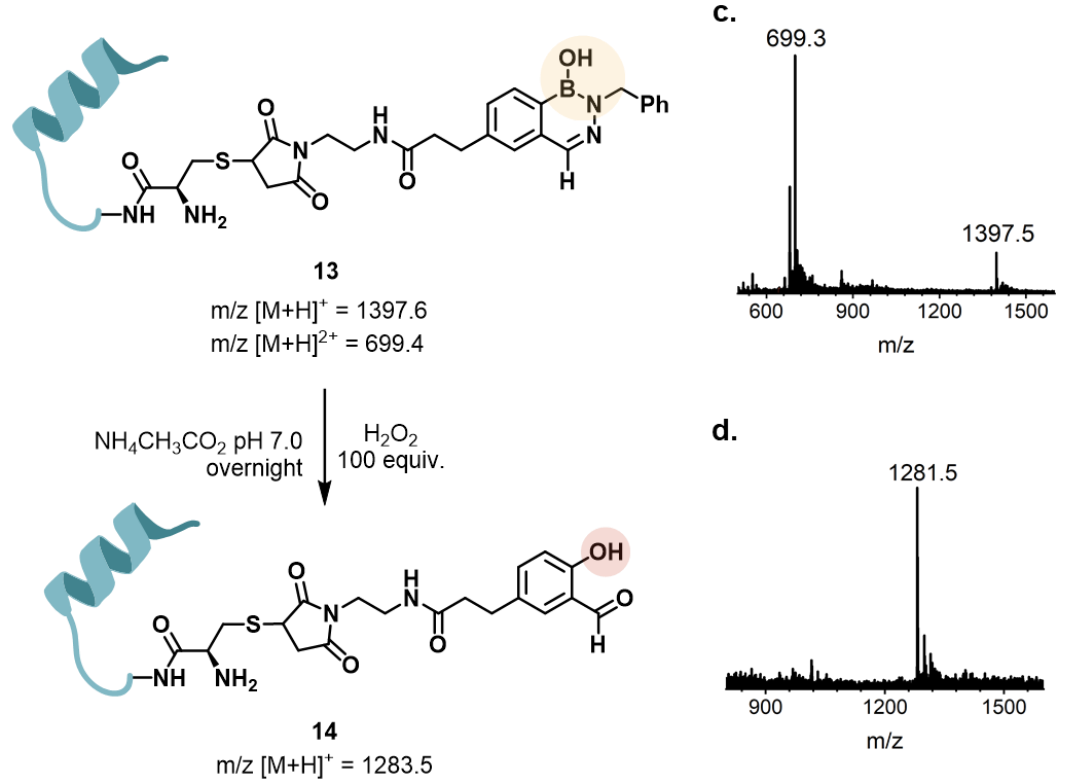

d.

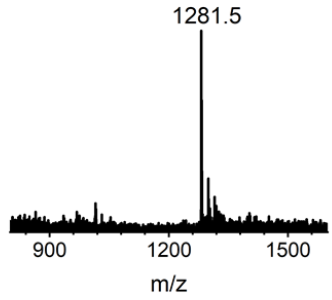

Figure 7 | Bioconjugation reaction between DAB 12 and Laminin fragment. (a) Model DAB 12, synthesized from 11 and benzyl hydrazine; (b) schematic representation of the conjugation between laminin and DAB 12 followed by oxidation with $\mathrm{H}_{2} \mathrm{O}_{2}$; (c) ESI-MS after bioconjugation; (d) ESI-MS in the presence of 100 equiv. $\mathrm{H}_{2} \mathrm{O}_{2}$ overnight. 
As shown in Fig. 7, the oxidation process also promoted the hydrolysis of the hydrazone. Based on this observation, we envisioned the possibility of exploiting this hydrolysis to trigger the release of a payload through a self-immolative mechanism. With this objective in mind, the strategies depicted in Fig. 8 were delineated to study if either the hydrazone or the salicylaldehyde components could act as selfimmolative modules. Considering the results previously obtained in Fig. 7, we first evaluated route A (Fig. 9). Hence, DABs 15 and $\mathbf{1 6}$ featuring a model benzyl alcohol were synthesized and evaluated in terms of stability and oxidation in the presence of $\mathrm{H}_{2} \mathrm{O}_{2}$ (KPi $50 \mathrm{mM}, \mathrm{pH}$ 7.4). Interestingly, DAB 15 proved to be quite unstable in these conditions, favoring the elimination of benzyl acrylate 18. On the other hand, DAB 16 was tested in the same conditions and exhibited a half-life of 9.2 days in KPi buffer at $\mathrm{pH}$ 7.4. More importantly, in the presence of $\mathrm{H}_{2} \mathrm{O}_{2}, \mathrm{DAB} \mathbf{1 6}$ was readily oxidized ( 80 min half-life) to generate salicylaldehyde 19, the hydrazone 20 and benzyl alcohol 21. Analyzing the evolution of the oxidation reaction (Fig. 9), upon addition of the oxidant, the concentration of salicyl hydrazone 19 rapidly increases, followed by a second stage characterized by a slow decrease in concentration of 19 (Fig. 9c). This kinetics profile suggests the oxidation step is faster than the hydrazone's hydrolysis to release benzyl alcohol 21.

a.

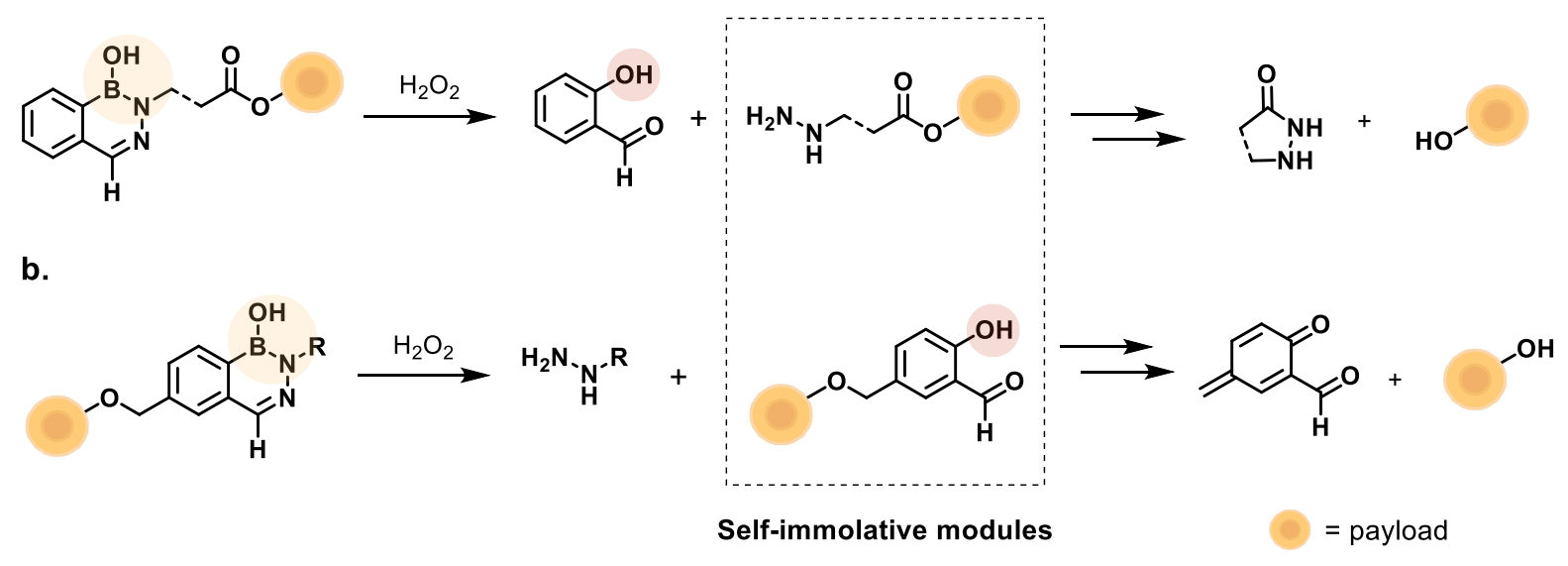

Figure 8 | Two strategies to design a triggered self-immolative release of a payload. The self-immolative module is incorporated (a) on the hydrazine moiety or (b) in the boronic acid/phenol module. 
a.<smiles>[Z4][C@H](C)CCCN1N=Cc2ccccc2B1O</smiles>

15

b.<smiles>O=C(CN1N=Cc2ccccc2B1O)OCc1ccccc1</smiles>

16

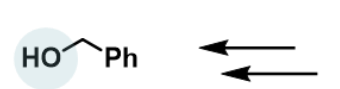

21
17<smiles>OB1NN=Cc2ccccc21</smiles><smiles>C=CC(=O)OCc1ccccc1</smiles>

18

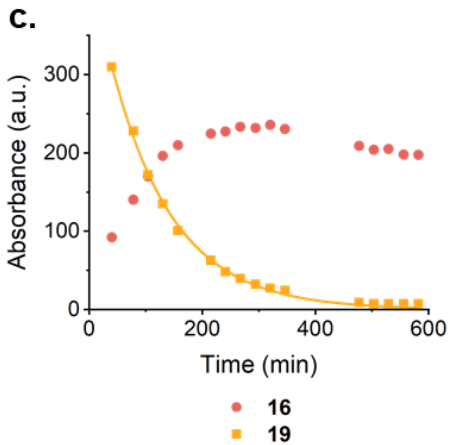

d.

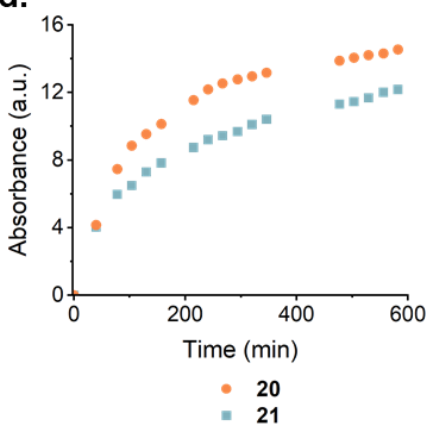

Figure 9 | DABs 15 and 16 featuring a model benzyl alcohol - stability and oxidation. (a) DAB 15 was shown to be unstable at pH 7.4; (b) DAB 16 is oxidized in the presence of 100 equiv. $\mathrm{H}_{2} \mathrm{O}_{2}$ and the hydrazine formed promotes the release of benzyl alcohol 21; (c) HPLC profiles of DAB 16 and the intermediate salicyl hydrazone 19 after incubation with 100 equiv. $\mathrm{H}_{2} \mathrm{O}_{2}$; (d) HPLC profiles of salicyl aldehyde 20 and benzyl alcohol 21 after incubation of DAB 16 with 100 equiv. $\mathrm{H}_{2} \mathrm{O}_{2}$.

Based on these results we next addressed the functionalization of the linker with the cytotoxic payload SN-38, a widely used cytotoxic agent in the clinic. Recently, SN-38 was used in the construction of an antibody drug conjugate (ADC - Sacituzumab govitecan - Trodelvy®) that was approved by FDA for the treatment of triple negative breast cancer. ${ }^{27-30}$ In our design, SN-38 was functionalized with the linker at the hydroxyl adjacent to the lactone ring, resulting in a esterification that generates a more stable construct. As shown in Fig. 10, compound 22 was reacted with the 2-FPBA component 11 to yield the DAB 23 that was used in the functionalization of the laminin fragment peptide. The bioconjugation reaction produced mainly bioconjugate $\mathbf{2 4}$, though careful analysis of the ESI-MS spectra indicated that the hydrolyzed conjugate was also being produced in small amounts (Supplementary Fig. S27). Considering this observation, we studied the stability of DAB 23 in PBS at $\mathrm{pH}$ 7.4. In these conditions, the compound exhibited a half-life of $8 \mathrm{~h}$, corroborating the observed hydrolysis of $\mathbf{2 4}$ (Supplementary Fig. S19). This slight instability is likely related to the presence of the ester function, which is known to be labile in aqueous conditions. 

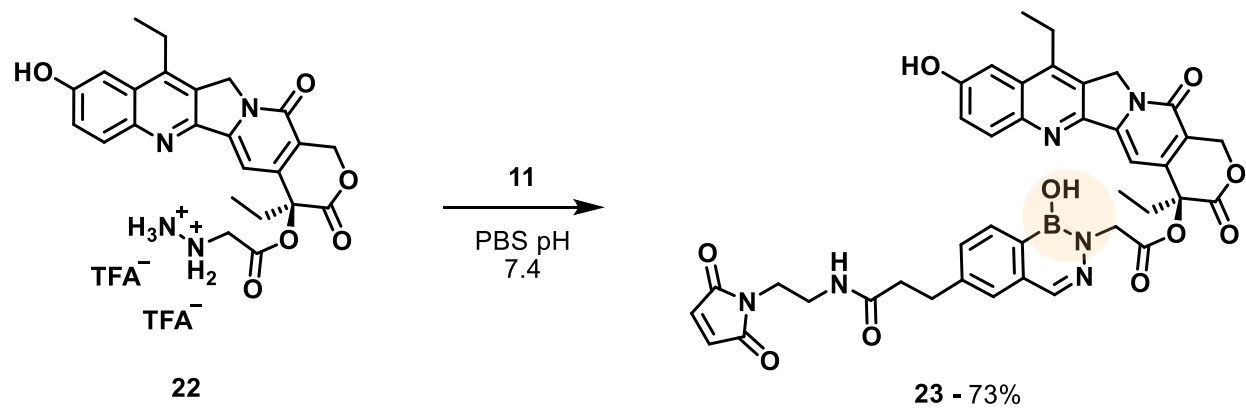

$23-73 \%$

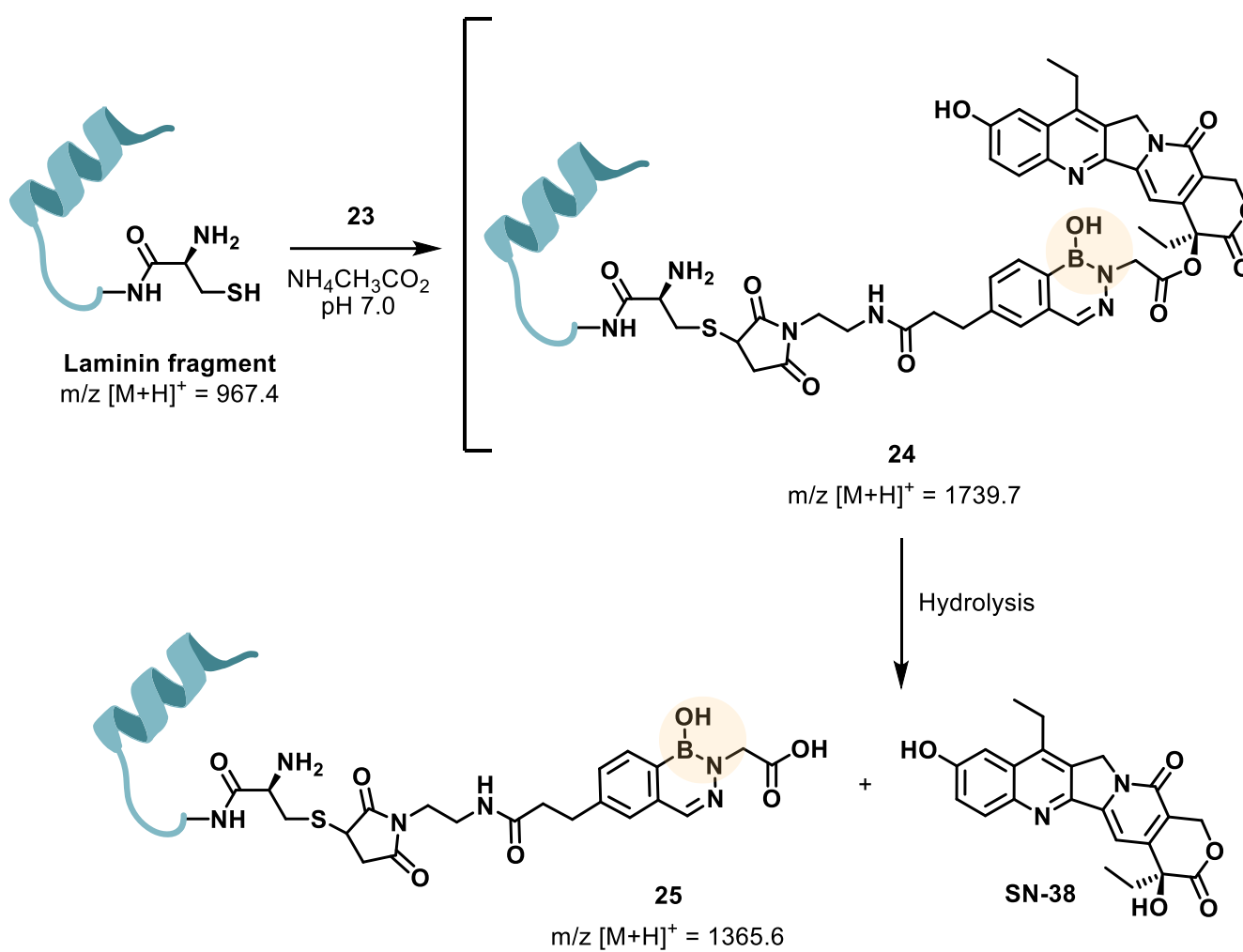

Figure 10 | Synthetic route for the preparation of linker-payload 23 and its use in the modification of a laminin fragment. The bioconjugation reaction was analysed by ESI-MS to confirm the presence of bioconjugate $\mathbf{2 4}$ and hydrolyzed conjugate $\mathbf{2 5}$ in small amounts. TFA, trifluoroacetic acid.

Considering the hydrolysis profile observed for $\mathrm{DAB} \mathbf{2 3}$, we evaluated if a self-immolative cascade directly promoted by the formation of the phenolic unit, would afford a more stable linker, as well as a more efficient release of the payload (Fig. 11). Therefore, starting from salicyladehyde, the 2-FPBA component was modified to include a benzyl alcohol para to the BA function, which then enabled the installation of SN-38, as shown in Fig. 11. 


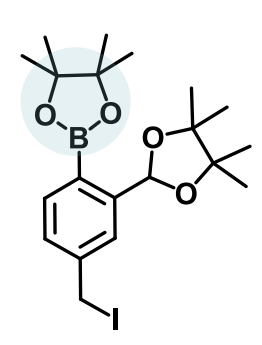

$26-54 \%$

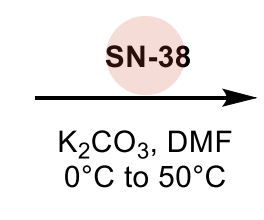

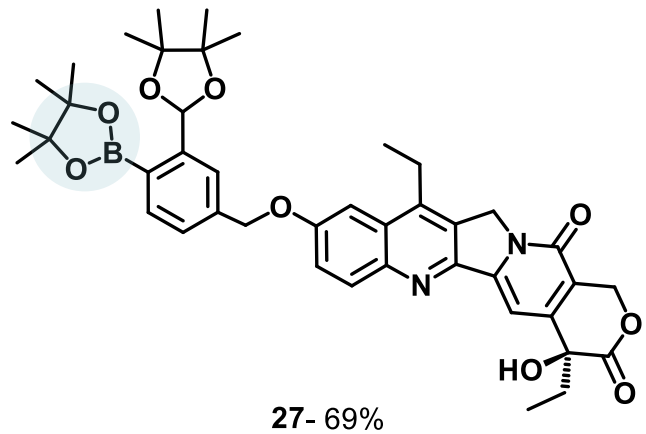

27- $69 \%$<smiles>CCc1c2c(nc3ccc(OCc4ccc5c(c4)C=NN(CC(=O)NCC(C)(C)OCC(C)(C)N)B5O)cc13)-c1cc3c(c(=O)n1C2)COC(=O)[C@]3(O)CC</smiles>

Figure 11 | Synthetic route for the preparation of linker-payload 29. Conditions for 27: compound 26 (1.5 equiv.), SN-38 (1 equiv.), $\mathrm{K}_{2} \mathrm{CO}_{3}$ (1.5 equiv.), DMF, $0^{\circ} \mathrm{C}-50^{\circ} \mathrm{C}$, 4h, 69\%. Conditions for 29: $\mathrm{HCl}$ in $\mathrm{H}_{2} \mathrm{O} / \mathrm{CH}_{3} \mathrm{CN}$ (4:3), $25^{\circ} \mathrm{C}, 24 \mathrm{~h}$; hydrazine 28 (6 equiv.) in $\mathrm{H}_{2} \mathrm{O} / \mathrm{CH}_{3} \mathrm{CN}$ (4:3), $25^{\circ} \mathrm{C}, 16 \mathrm{~h}, 39 \%$. DMF, N,N-dimethylformamide.

Once prepared, compound $\mathbf{2 7}$ was reacted with hydrazine $\mathbf{2 8}$ to afford DAB $\mathbf{2 9}$. This hydrazine $\mathbf{2 8 ,}$ featuring a small polyethylene glycol, was selected to improve the aqueous solubility of the linker and to enable its installation on the biomolecule surface by a strain-promoted azide-alkyne cycloaddition (SPAAC) reaction. Once prepared, DAB 29 was tested for its stability in PBS pH 7.4 where it displayed a half-life of over 100h (Fig.12a). Similarly, a high stability was also observed for this compound in human plasma (Fig.12b). Nonetheless, by adding $\mathrm{H}_{2} \mathrm{O}_{2}$ to 29 in $\mathrm{PBS}$ at $\mathrm{pH} 7.4$, the diazaborine was readily oxidized (half-life $8.1 \mathrm{~h}$ ) and released the SN-38 drug (Fig. 12c-d). 
a.

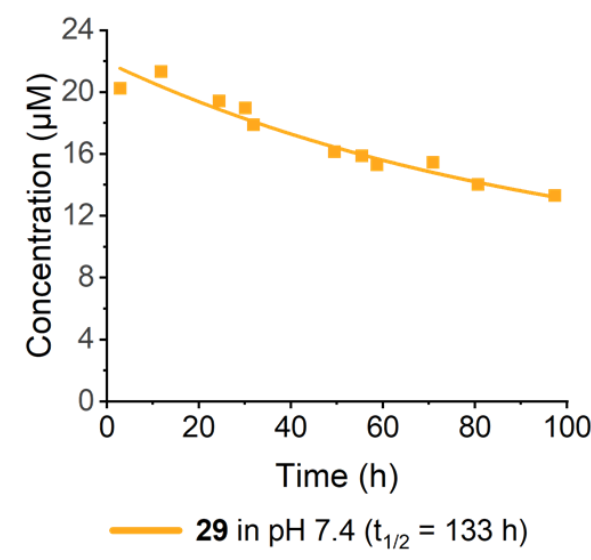

C.

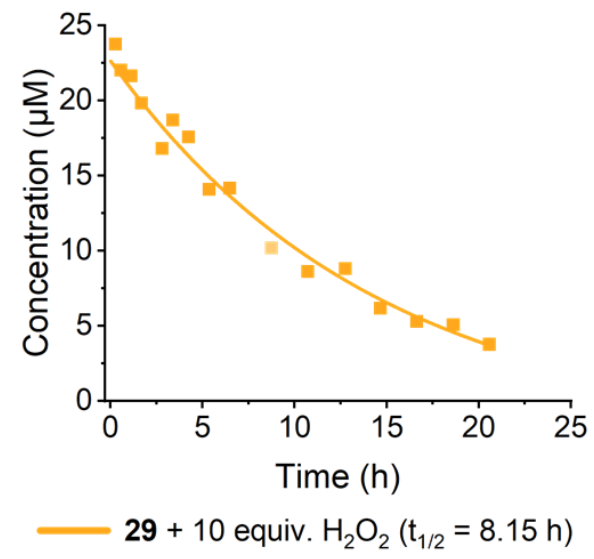

b.

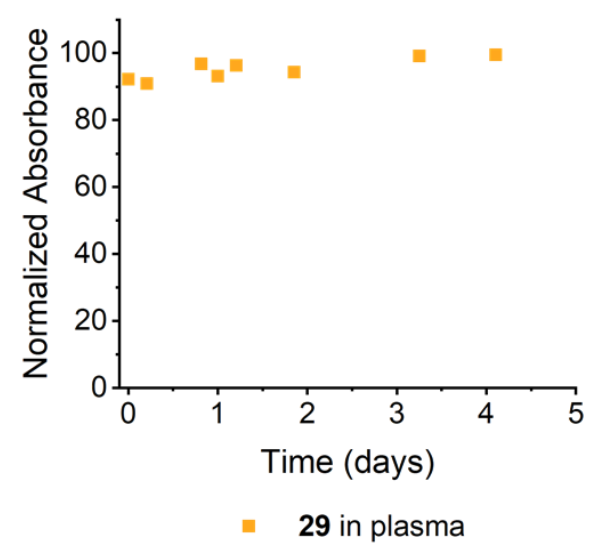

d.

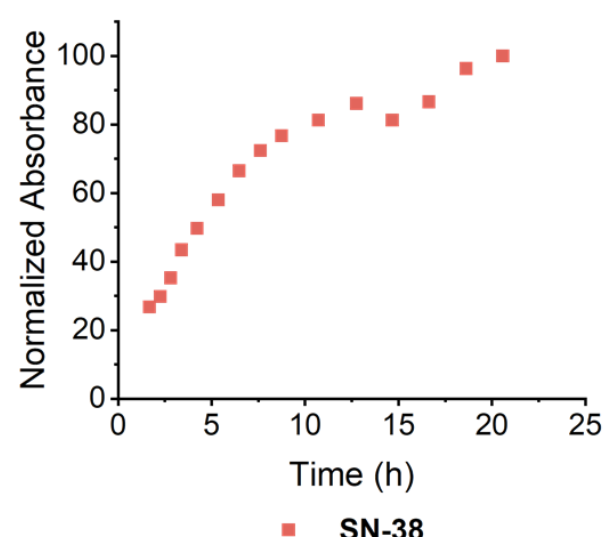

Figure 12 | DAB 29 stability at different conditions analysed by HPLC. (a) DAB 29 stability in PBS/DMSO pH 7.4 (b) DAB 29 stability in plasma; (c) Oxidation profile profile of DAB 29 oxidation in the presence of 100 equiv $\mathrm{H}_{2} \mathrm{O}_{2}$ (disappearance of the starting material) in PBS/DMSO pH 7.4; (d) SN-38 release profile during DAB 29 oxidation in the presence of 100 equiv. $\mathrm{H}_{2} \mathrm{O}_{2}$ in PBS/DMSO pH 7.4. DMF, n,n-dimethylformamide.

Then, we studied the conjugation of DAB 29 to the laminin fragment. To this end, a commercially available maleimide-cyclooctyne was used to functionalize the peptide and to react with DAB 29 by a SPAAC reaction to generate bioconjugate Laminin-DAB31-SN-38 (Fig. 13). This reaction effectively generated the expected bioconjugate $\mathbf{3 1}$, thereby a similar methodology was employed to construct a functional ADC against cancer cells. 

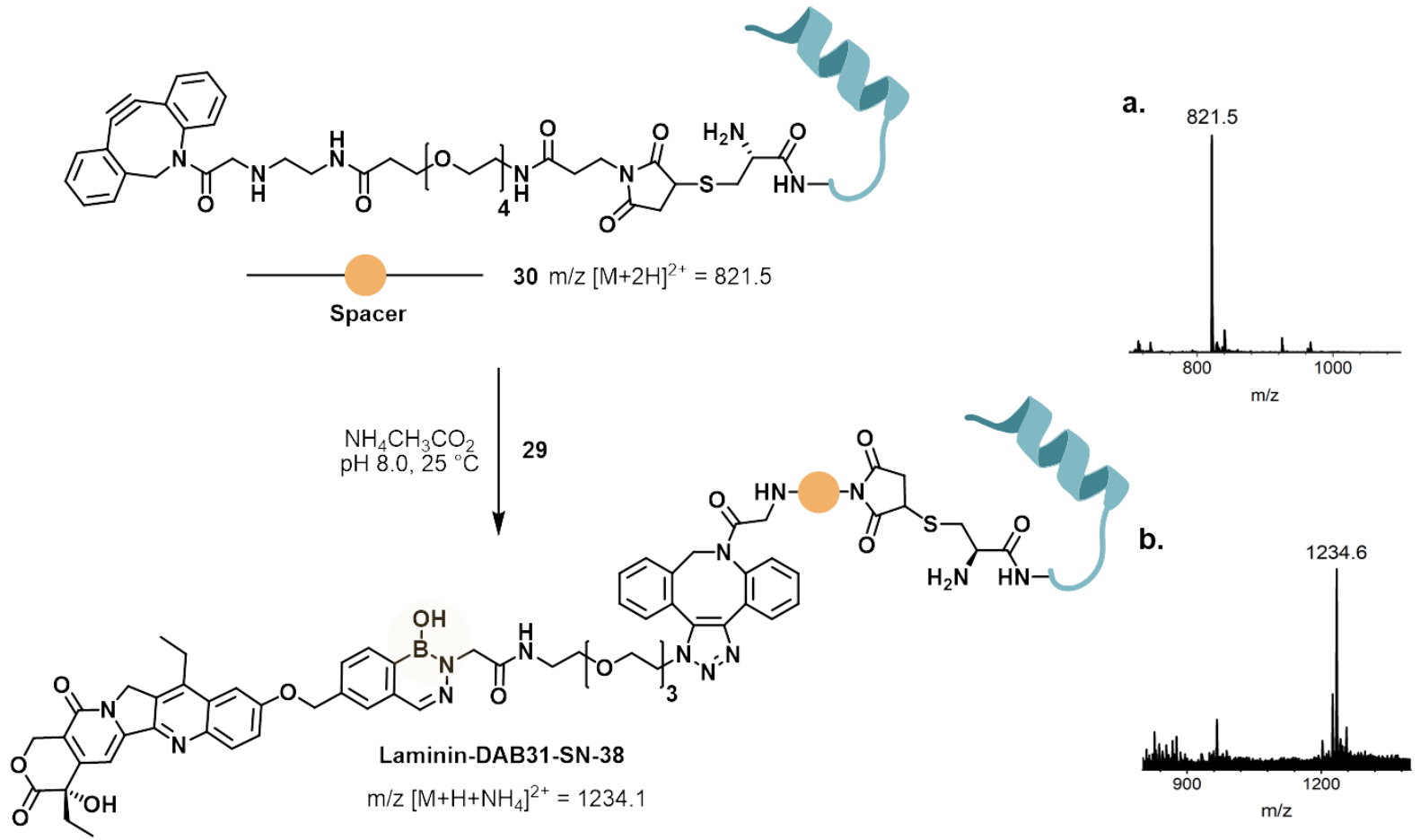

Figure 13 | Reaction of DAB 29 in DMSO ( $2 \mathrm{mM})$ with laminin in ammonium acetate solution $(10 \mu \mathrm{M}, 20$ mM, pH 8) at room temperature, after adding commercially available maleimide-PEG-cyclooctyne. (a) ESIMS spectrum after the reaction of laminin with maleimide-PEG-cyclooctyne; (b) ESI-MS spectrum after the addition of compound 29.

Recently, we initiated a program to develop an antibody fragment that selectively targets B-cell lymphoma cells. This antibody scaffold consists on a rabbit derived VL single-domain antibody that exhibits a free cysteine at 82 position and can be explored to develop antibody-drug conjugates (ADC). This antibody scaffold was therefore modified with the maleimide-cyclooctyne cross linker and converted in the homogenous targeting drug conjugate 31, VL-DAB31-SN-38, using the DAB 29 linker (Fig. 14). 

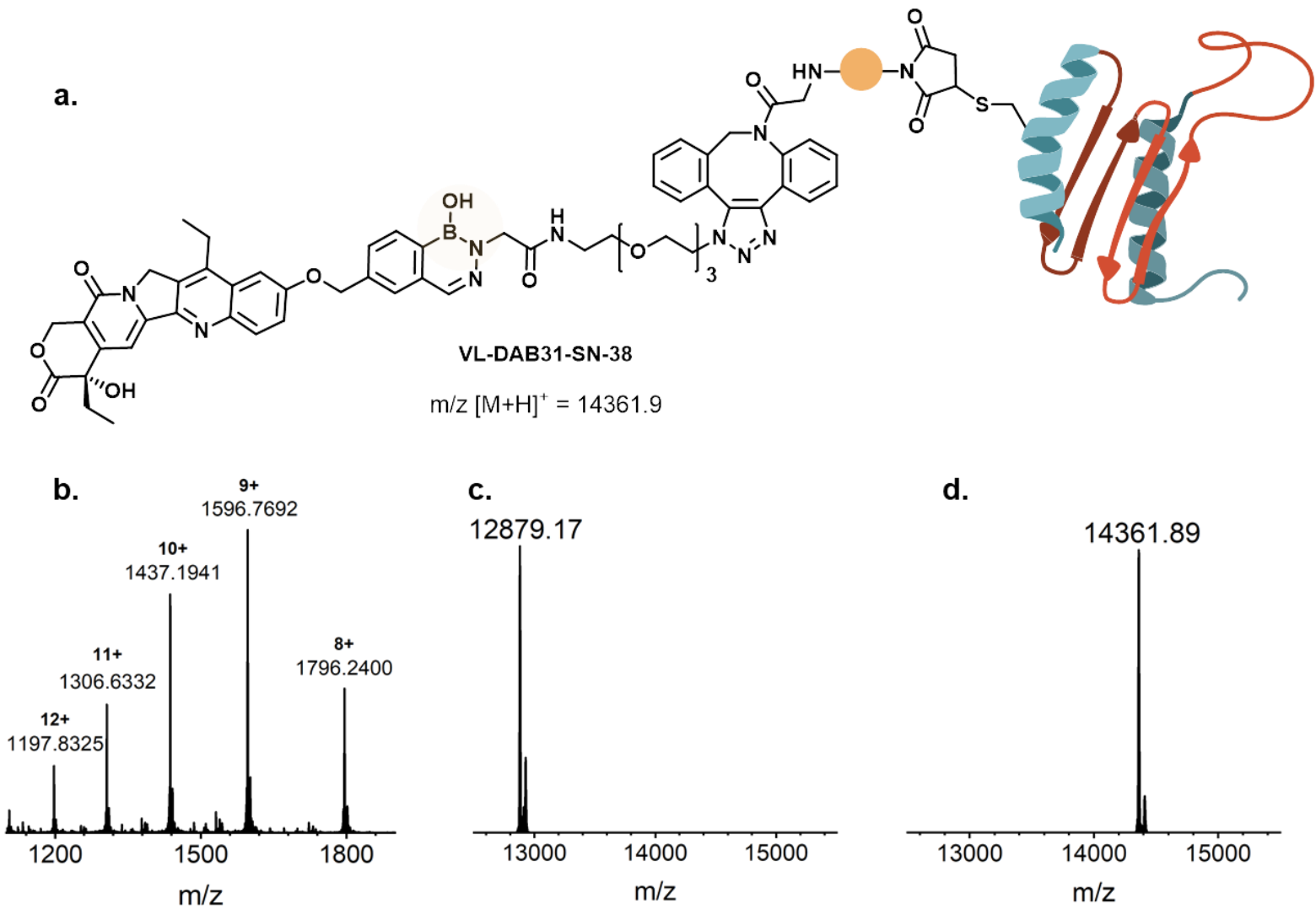

c.

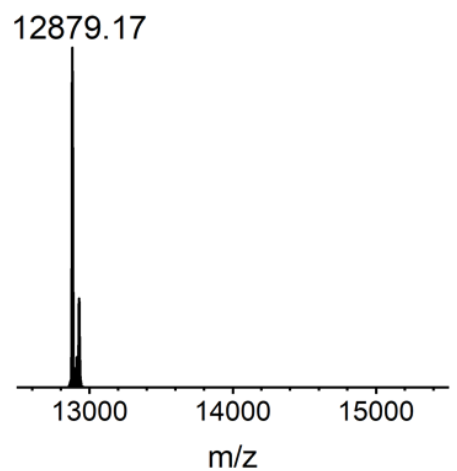

d.

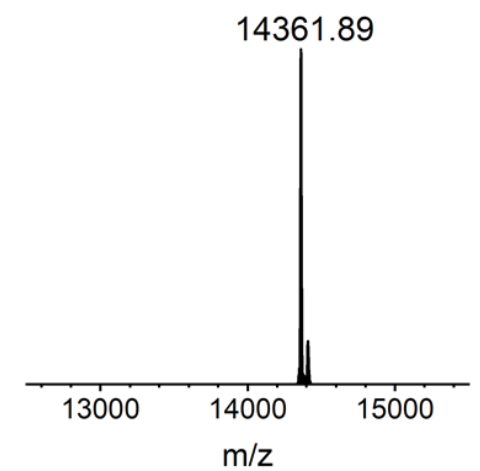

Figure 14 | Conjugate VL-DAB31-SN-38 obtained from VL's cysteine modification with commercially available cyclooctyne 30, followed by a SPAAC with DAB 29 (more details in the Supporting Info). (a) HRMS spectrum of conjugate VL-DAB31-SN-38; (b) Deconvoluted HRMS spectrum of VL (Mass: 12879.2 Da); (c) Deconvoluted HRMS spectrum of conjugate VL-DAB31-SN-38 (14361.9 Da). Mass spectral deconvolution was performed using Zscore algorithm in MagTran1.03 software. ${ }^{31}$

Once prepared, this ADC was tested in the canine B-cell lymphoma CLBL-1 cell line and Jurkat cell line (control). The conjugate VL-DAB31-SN-38 revealed to be highly cytotoxic toward the CLBL-1 cancer cell line with an $\mathrm{IC}_{50}$ value of $54.1 \mathrm{nM}$ (Fig. 15a). In contrast, the unconjugated VL did not show cytotoxicity under the same conditions. Additionally, conjugate VL-DAB31-SN-38 did not present a cytotoxic activity against the Jurkat control cell line (T lymphocytes) (Fig. 15b). These results indicated that the conjugate must be internalized to elicit its cytotoxic activity. 
a.

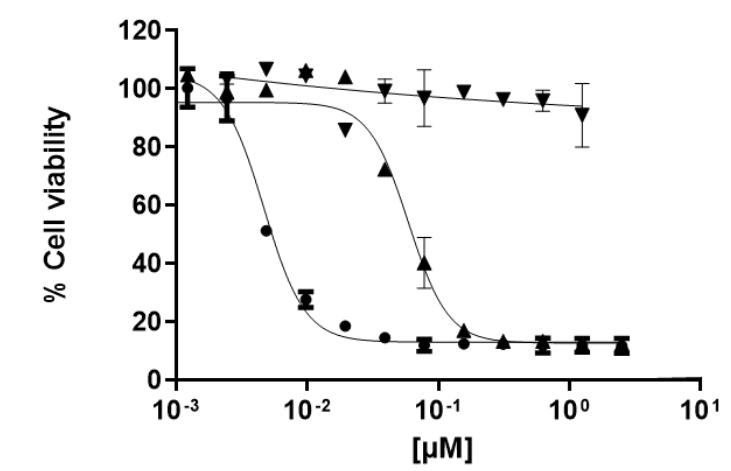

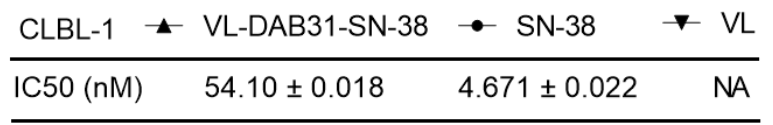

b.

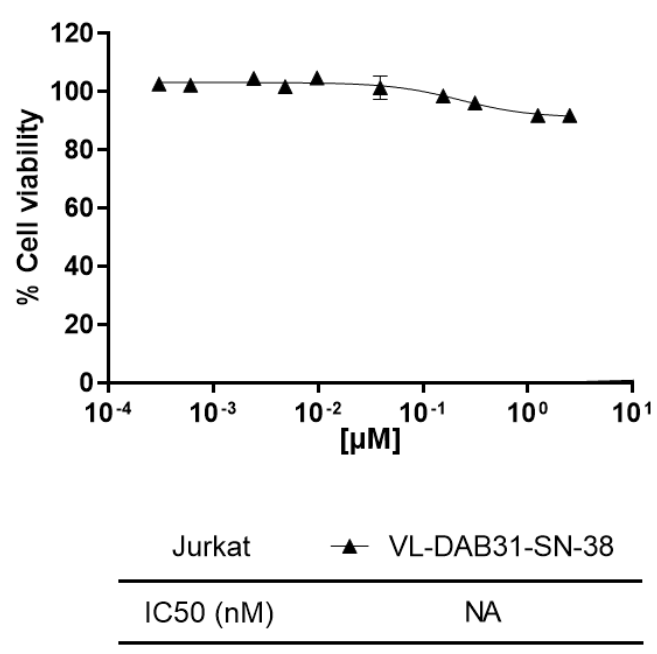

Figure 15 | Cytotoxicity of conjugate VL-DAB31-SN-38. (a) Cytotoxic effect of conjugate VL-DAB31-SN-38 on the CLBL-1 cell line. CLBL-1 cells $\left(6 \times 10^{4}\right)$ were subjected to the indicated concentrations of VL-DAB31SN-38, SN-38 and VL. After $48 \mathrm{~h}$ treatment, cell viability and proliferation were evaluated with WST-1 reagent. Best-fit $\mathrm{IC}_{50}$ values of each compound were calculated using the log (inhibitor) vs response (variable slope) function; (b) Viability assay for conjugate VL-DAB31-SN-38 on the Jurkat cell line (incubation time of $48 \mathrm{~h}$ ).

\section{Conclusions}

In summary, here we presented an innovative technology to synthesize ROS-responsive ADCs based on DABs. The high stability of DAB 2 in buffer (over 14 days at $\mathrm{pH} 4.5,7.4$ and 9; plasma) and in plasma (over 5 days), together with the ability to be oxidized in the presence of $\mathrm{H}_{2} \mathrm{O}_{2}\left(0.422\right.$ and $0.103 \mathrm{M}^{-}$ ${ }^{1} \mathrm{~S}^{-1}$ with 100 and 10 equiv. of $\mathrm{H}_{2} \mathrm{O}_{2}$ respectively) made this scaffold a very useful tool to prepare welldefined ROS-responsive ADCs. A detailed DFT study was performed to elucidate the mechanism of DAB 2 oxidation in the presence of $\mathrm{H}_{2} \mathrm{O}_{2}$, which revealed a similar pathway to the known oxidation of aromatic boronic acids with $\mathrm{H}_{2} \mathrm{O}_{2}$. Once established the high stability and ROS responsiveness of DABs, these scaffolds were applied in the synthesis of self-immolative linkers to release the cytotoxic payload SN-38. The linker was equipped with a maleimide that was used in the site-selective functionalization of a VL antibody. The obtained ADC VL-DAB31-SN-38 presented a high selectivity and potency $\left(\mathrm{IC}_{50}=54.1 \mathrm{nM}\right)$ against B-cell lymphoma CLBL-1 cell line. 
ROS is essential to sustain the biochemical alterations required for the initiation, promotion and progression of cancer, but is also a distinctive feature of many other important diseases (e.g neurodegeneration and inflammation), and until now, the development of ROS-responsive ADCs for these conditions has been limited by the existence of stable linkers that can effectively respond to ROS. The developed DABs oxidation technology is expected to overcome these limitations, and support the discovery of the next generation of stimuli-responsive ADCs.

\section{Present Addresses}

$\dagger$ If an author's address is different than the one given in the affiliation line, this information may be included here.

\section{Author Contributions}

The manuscript was written through contributions of all authors. / All authors have given approval to the final version of the manuscript. / $\ddagger$ These authors contributed equally. (match statement to author names with a symbol)

\section{ACKNOWLEDGMENT}

The authors acknowledge the financial support from Fundação para a Ciência e a Tecnologia (FCT), Ministério da Ciência e da Tecnologia, Portugal (SFRH/BD/90514/2012 PD/BD/128239/2016, PD/BD/143124/2019, $\quad$ SFRH/BPD/102296/2014, iMed.ULisboa $\quad$ UIDB/04138/2020; SAICTPAC/0019/2015, PTDC/QUI-QOR/29967/2017, PTDC/BTM-SAL/32085/2017); LISBOA-010145-FEDER-029967. (LFV) Centro de Química Estrutural acknowledges the financial support of FCT (UIDB/00100/2020).

\section{ABBREVIATIONS}


2-APBA, 2-acetylphenyl boronic acid; 2-FPBA, 2-formylphenyl boronic acid; ADC, Antibody-drug conjugate; BA, boronic acid; CLBL-1, canine B-cell lymphoma cell line; DAB, diazaborine; DFT, density functional theory; DMSO; dimethyl sulfoxide; ESI-MS, electrospray ionization - mass spectrometry; FDA, Food and Drug Administration; GSH, glutathione; HPLC, high performance liquid chromatography; $\mathrm{IC}_{50}$, half-maximal inhibitory concentration; LC-HRMS, liquid chromatography-high resolution mass spectrometry; PBS, phosphate-buffered saline; SPAAC, azide-alkyne cycloaddition; ROS, reactive species of oxygen; TFA, trifluoroacetic acid; VL, light-chain variable region.

\section{REFERENCES}

1. Sabharwal, S. S. \& Schumacker, P. T. Mitochondrial ROS in cancer: initiators, amplifiers or an Achilles' heel? Nat. Rev. Cancer 14, 709-721 (2014).

2. Liou, G. Y. \& Storz, P. Reactive oxygen species in cancer. Free Radical Research vol. 44 (2010).

3. Reczek, C. R. \& Chandel, N. S. The Two Faces of Reactive Oxygen Species in Cancer. Annu. Rev. Cancer Biol. 1, 79-98 (2017).

4. Bansal, A. \& Simon, M. C. Glutathione metabolism in cancer progression and treatment resistance. J. Cell Biol. 217, 2291-2298 (2018).

5. Lv, H. et al. Unraveling the Potential Role of Glutathione in Multiple Forms of Cell Death in Cancer Therapy. Oxid. Med. Cell. Longev. 2019, 1-16 (2019).

6. Gorrini, C., Harris, I. S. \& Mak, T. W. Modulation of oxidative stress as an anticancer strategy. Nat. Rev. Drug Discov. 12, 931-947 (2013).

7. Liang, J. \& Liu, B. ROS-responsive drug delivery systems. Bioeng. Transl. Med. 1, 239-251 (2016).

8. Xu, X. et al. ROS-Responsive Polyprodrug Nanoparticles for Triggered Drug Delivery and Effective Cancer Therapy. Adv. Mater. 29, 1-6 (2017). 
9. Dong, Z., Yang, Z., Hao, Y. \& Feng, L. Fabrication of H2O2 -driven nanoreactors for innovative cancer treatments. Nanoscale 11, 16164-16186 (2019).

10. Schmidt, P., Stress, C. \& Gillingham, D. Boronic acids facilitate rapid oxime condensations at neutral pH. Chem. Sci. 6, 3329-3333 (2015).

11. Kim, E. J. et al. An activatable prodrug for the treatment of metastatic tumors. J. Am. Chem. Soc. 136, 13888-13894 (2014).

12. António, J. P. M., Russo, R., Carvalho, C. P., Cal, P. M. S. D. \& Gois, P. M. P. Boronic acids as building blocks for the construction of therapeutically useful bioconjugates. Chem. Soc. Rev. 48, 3513-3536 (2019).

13. Stubelius, A., Lee, S. \& Almutairi, A. The Chemistry of Boronic Acids in Nanomaterials for Drug Delivery. Acc. Chem. Res. 52, 3108-3119 (2019).

14. Högenauer, G. \& Woisetschläger, M. A diazaborine derivative inhibits lipopolysaccharide biosynthesis. Nature 293, 662-664 (1981).

15. Grassberger, M. A., Turnowsky, F. \& Hildebrandt, J. Preparation and antibacterial activities of new 1,2,3-diazaborine derivatives and analogs. J. Med. Chem. 27, 947-953 (1984).

16. Baldock, C. et al. A mechanism of drug action revealed by structural studies of Enoyl reductase. Science (80-. ). 274, 2107-2110 (1996).

17. Baldock, C., De Boer, G. J., Rafferty, J. B., Stuitje, A. R. \& Rice, D. W. Mechanism of Action of Diazaborines. Biochem. Pharmacol. 55, 1541-1550 (1998).

18. Bandyopadhyay, A., Cambray, S. \& Gao, J. Fast Diazaborine Formation of Semicarbazide Enables Facile Labeling of Bacterial Pathogens. J. Am. Chem. Soc. 139, 871-878 (2017).

19. Chio, T. I., Gu, H., Mukherjee, K., Tumey, L. N. \& Bane, S. L. Site-Specific Bioconjugation and Multi-Bioorthogonal Labeling via Rapid Formation of a Boron-Nitrogen Heterocycle. Bioconjug. Chem. 30, 1554-1564 (2019). 
20. António, J. P. M., Gonçalves, L. M., Guedes, R. C., Moreira, R. \& Gois, P. M. P. Diazaborines as New Inhibitors of Human Neutrophil Elastase. ACS Omega 3, 7418-7423 (2018).

21. Kazmi, M. Z. H. et al. Lewis or Brønsted? A Rectification of the Acidic and Aromatic Nature of Boranol-Containing Naphthoid Heterocycles. J. Am. Chem. Soc. jacs.1c02462 (2021) doi:10.1021/jacs.1c02462.

22. Dilek, O., Lei, Z., Mukherjee, K. \& Bane, S. Rapid formation of a stable boron-nitrogen heterocycle in dilute, neutral aqueous solution for bioorthogonal coupling reactions. Chem. Commun. 51, 16992-16995 (2015).

23. Kanichar, D. et al. Synthesis, Characterization, and Antibacterial Activity of Structurally Complex 2-Acylated 2,3,1-Benzodiazaborines and Related Compounds. Chem. Biodivers. 11, 1381-1397 (2014).

24. Parr, R. G. \& Yang, W. Density Functional Theory of Atoms and Molecules. Density Functional Theory of Atoms and Molecules (1989).

25. Kuivila, H. G. \& Armour, A. G. Electrophilic Displacement Reactions. IX. Effects of Substituents on Rates of Reactions between Hydrogen Peroxide and Benzeneboronic Acid 1-3. J. Am. Chem. Soc. 79, 5659-5662 (1957).

26. Kuivila, H. G. \& Muller, T. C. Electrophilic Displacement Reactions. XIII. the Kinetics of the Phenylmercurideboronation of Benzeneboronic Acid 1-3. J. Am. Chem. Soc. 84, 377-382 (1962).

27. Goldenberg, D. M. \& Sharkey, R. M. Sacituzumab govitecan, a novel, third-generation, antibody-drug conjugate (ADC) for cancer therapy. Expert Opin. Biol. Ther. 20, 871-885 (2020).

28. Nagayama, A., Vidula, N., Ellisen, L. \& Bardia, A. Novel antibody-drug conjugates for triple negative breast cancer. Ther. Adv. Med. Oncol. 12, 175883592091598 (2020). 
29. Lopez, S. et al. Preclinical activity of sacituzumab govitecan (IMMU-132) in uterine and ovarian carcinosarcomas. Oncotarget 11, 560-570 (2020).

30. Goldenberg, D. M., Cardillo, T. M., Govindan, S. V., Rossi, E. A. \& Sharkey, R. M. Trop-2 is a novel target for solid cancer therapy with sacituzumab govitecan (IMMU-132), an antibodydrug conjugate (ADC). Oncotarget 11, 942-942 (2020).

31. Zhang, Z. \& Marshall, A. G. A universal algorithm for fast and automated charge state deconvolution of electrospray mass-to-charge ratio spectra. J. Am. Soc. Mass Spectrom. 9, 225233 (1998). 